\title{
Polar-surface-driven growth of ZnS microsprings with novel optoelectronic properties
}

\author{
Qi Zhang ${ }^{1}$, Cong $\mathrm{Wei}^{2}$, Xing $\mathrm{Li}^{3}$, Muhammad Hafeez ${ }^{1}$, Lin Gan ${ }^{1}$, Huiqiao $\mathrm{Li}^{1}$, Xianlong Wei ${ }^{3}$, \\ Yongsheng $\mathrm{Zhao}^{2}$, Ying $\mathrm{Ma}^{1}$ and Tianyou Zhai ${ }^{1}$
}

Small perturbations during the growth kinetics of low-dimensional semiconductor structures may lead to novel features, which may provide an ideal system for understanding the fundamental physics of optical phenomena in optoelectronics devices. In this study, we report deformation-free single-crystal zinc sulfide $(\mathrm{ZnS})$ microsprings produced by a polar-surface-driven growth process, and we thoroughly investigate the electrical characteristics of individual ZnS microsprings under electron beam irradiation and their initial applications in ultraviolet (UV) light sensors and waveguides. The microsprings produced are formed by a block-by-block stacking process following a hexagonal screw model that does not introduce distortion into the crystal lattices. The first photodetectors designed based on a single $\mathrm{ZnS}$ microspring exhibit a high spectral selectivity combined with a high photosensitivity and a fast response time $(<0.3 \mathrm{~s})$ under $320-\mathrm{nm}$ illumination, which make $\mathrm{ZnS}$ microsprings particularly valuable as UV-light sensors. Concurrently, excellent waveguide performance along the fiber of a single $\mathrm{ZnS}$ microspring (for

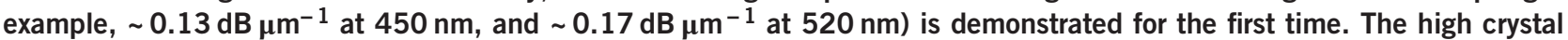
quality, the fast response to UV light and the low propagation loss exhibited by $\mathrm{ZnS}$ microsprings indicate that they have important potential applications in nano-optoelectronic systems. NPG Asia Materials (2015) 7, e213; doi:10.1038/am.2015.100; published online 4 September 2015

\section{INTRODUCTION}

One-dimensional semiconductor structures provide new opportunities to exploit the chemical and physical properties of materials at the micro- and nano-scales, making them promising candidates for future optoelectronic devices that take advantage of the well-controlled size, morphology and geometries of these materials. ${ }^{1,2}$ The most recent studies have shown that bent nanowires or nanobelts can exhibit novel optical and electrical properties compared with their unbent counterparts, thus appreciably widening their potential applications in optoelectronic systems because optoelectronic characteristics in lowdimensional materials are particularly sensitive to crystallinity and electronic structures. ${ }^{3}$ For example, in the photoluminescence spectra of bending wurtzite micro/nanowires, a red shift of the near-band energy induced by deformation has been shown to have a linear relation between the peak shift and the strain gradient. ${ }^{4}$ Because the peaks of the photoluminescence spectra are near the band gap of these materials, such a linear correlation between the band gap and strain gradient could provide an alternative method in optoelectronics, such as enhanced photodetectors. ${ }^{5}$ Conversely, from both scientific and practical viewpoints, one-dimensional structures are ideal materials to measure the optical gains of miniaturized lasers and light amplification due to their strong ability to confine electrons, holes and photons.
However, in straight cavities such as micro/nanowires, travelling photons are primarily scattered at the tips, ${ }^{6}$ which severely degrades the waveguide performance. To address this issue, Tong and coworkers ${ }^{7}$ proposed a helical cavity that can significantly promote the out-coupling of an emission and provide a higher power output than from other types of straight cavities. Even for organic helical structures, Zhao and coworkers ${ }^{8}$ confirmed that they could confine fluorescence to achieve whispering gallery mode resonance, supporting the novel optical properties of helical structures for optoelectronic systems. To date, a large number of helical or spring semiconductor materials have been produced via perturbations in the growth kinetics. ${ }^{9,10}$ Zinc sulfide (ZnS) micro/nanoscale structures have attracted considerable attention due to the size-dependent tuning of their functional properties and thus have applications in diverse photonics fields such as light-emitting diodes, lasers and ultraviolet (UV) light sensors. ${ }^{11,12}$ Considering the benefits of helical structures, it would thus be of particular interest to synthesize $\mathrm{ZnS}$ materials with helical structure and investigate their novel properties for optoelectronic systems. ${ }^{13}$

In this article, we report on the fabrication of high-quality, singlecrystal $\mathrm{ZnS}$ microsprings via a simple vapor deposition process and explore their potential applications in optoelectronic devices for the

${ }^{1}$ State Key Laboratory of Material Processing and Die \& Mould Technology, School of Materials Science and Engineering, Huazhong University of Science and Technology (HUST), Wuhan, China; ${ }^{2}$ Beijing National Laboratory for Molecular Sciences (BNLMS), CAS Key Laboratory of Photochemistry, Institute of Chemistry, Chinese Academy of Sciences (ICCAS), Beijing, China and ${ }^{3}$ Key Laboratory for the Physics and Chemistry of Nanodevices, Department of Electronics, Peking University, Beijing, China

Correspondence: Professor T Zhai, State Key Laboratory of Material Processing and Die \& Mould Technology, School of Materials Science and Engineering, Huazhong University of Science and Technology, 1037 Luoyu Road, Wuhan 430074, China.

E-mail: zhaity@hust.edu.cn

Received 15 April 2015; revised 28 May 2015; accepted 21 June 2015 

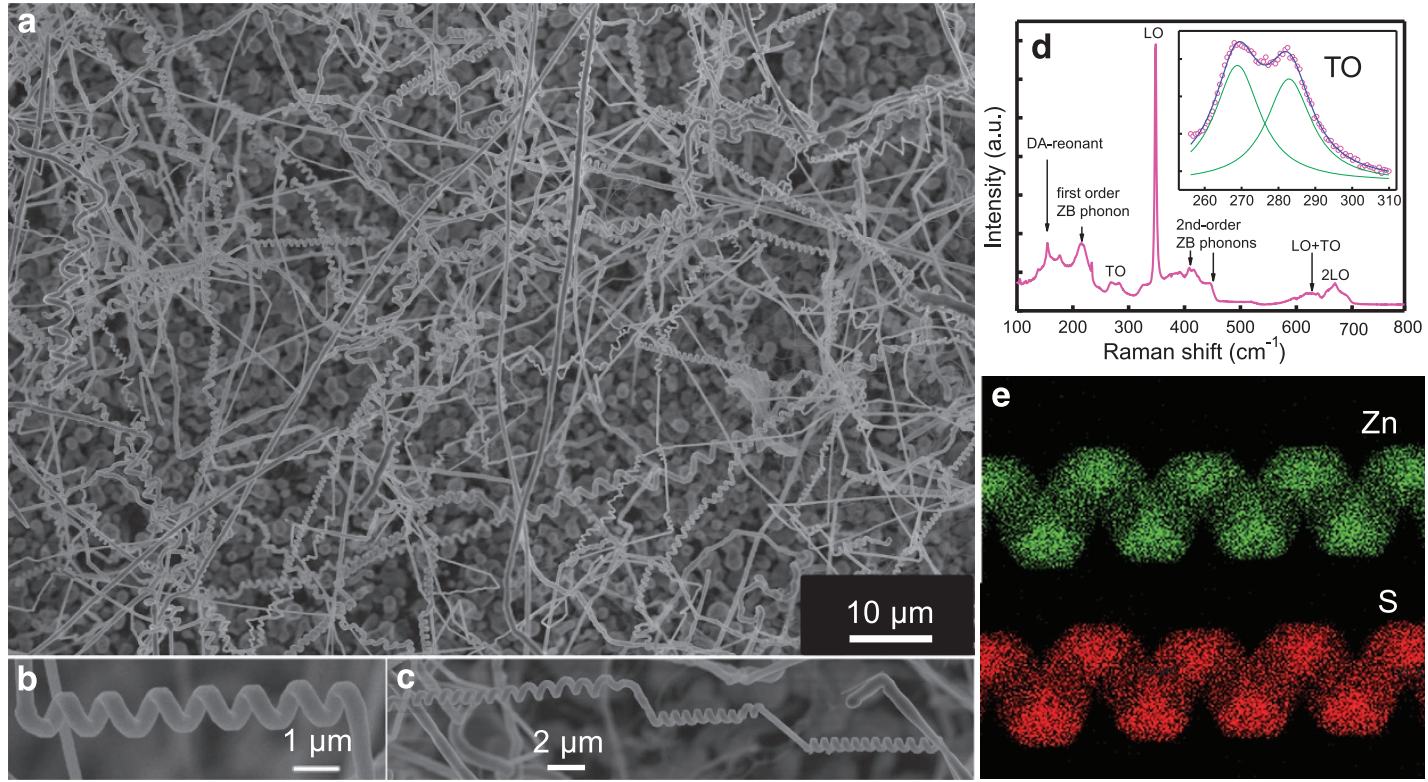

Figure 1 (a) Low-magnification SEM image of the as-synthesized ZnS microsprings, showing large-scale production; (b) enlarged image of a typical ZnS microspring; (c) coexistence of left- and right-handed chirality in an individual ZnS microspring; (d) Raman spectrum of ZnS springs collected in an atmospheric environment with an inset that shows the Lorentzian-line shape analysis of the TO doublet of $269,282 \mathrm{~cm}^{-1}$; and (e) EDS mapping of a single spring with uniform element distributions. 2D, two dimensional; a.u., arbitrary unit; DA, disorder activated; EDS, energy-dispersive spectroscopy; LO, longitudinal optical; SEM, scanning electron microscope; TO, transverse optical; ZB, zone boundary.

first time. The formation of $\mathrm{ZnS}$ springs is dominated by a change in the growth direction of $\langle 0-111\rangle$ that has six equivalent directions following a screw-coiling model. The electron beam irradiation (EBI)dependent electrical properties of individual $\mathrm{ZnS}$ springs were investigated in situ with a given mechanism, and a significant positive correlation between the electron energy and the internal gain was observed. We designed the first $\mathrm{ZnS}$ microspring based photodetectors with a good stability and a fast response time under a vertically illuminated UV light. In addition, the light confinement performance in individual $\mathrm{ZnS}$ springs was investigated, and the light intensity shows an exponential decay with propagation distance and a lowpropagation loss. These findings suggest a significant potential for using these novel $\mathrm{ZnS}$ springs in future optoelectronic devices.

\section{EXPERIMENTAL PROCEDURES}

\section{Synthesis and characterization of $\mathrm{ZnS}$ microsprings}

Si substrates in the (100) direction were used for growing the springs of interest in this study. Manganese (Mn)-catalyst thin films were deposited by resistive evaporation in an ultrahigh vacuum chamber at a pressure of $10^{-7}$ Torr at room temperature. The $\mathrm{Mn}$-coated $\mathrm{Si}$ substrates were then placed in a horizontal tube furnace at $850^{\circ} \mathrm{C} . \mathrm{ZnS}$ powder $(99.99 \% 0.5 \mathrm{~g})$ was placed in an alumina boat and was heated to $1100^{\circ} \mathrm{C}$. The carrier gas $(95 \%$ nitrogen and $5 \%$ hydrogen) flowed through the tube at a rate of 20 s.c.c.m. for $2 \mathrm{~h}$ before the furnace was switched off. The as-prepared $\mathrm{ZnS}$ springs were analyzed by fieldemission scanning electron microscopy (SEM; S-4800, Hitachi, Tokyo, Japan) and transmission electron microscopy (TEM; JSM-2010F, JEOL, Tokyo, Japan) with X-ray energy-dispersive spectroscopy. The Raman and optical properties of the samples were studied using a spectrometer (Horiba Jobin-Yoon HR800, Tokyo, Japan).

\section{Electrical properties measured by EBI}

The EBI-dependent electrical properties of the individual $\mathrm{ZnS}$ microsprings were performed in situ in an scanning electron microscope (SEM; Quanta 600F, FEI, Hillsboro, OR, USA) that was equipped with manipulators connected to a semiconductor parameter analyzer (Keithley 4200-SCS, Cleveland, OH, USA).

\section{Propagation properties of the $\mathrm{ZnS}$ microsprings}

The waveguide behavior of the samples was investigated using a microscope (Nikon Ti-U, Tokyo, Japan). The Ar-ion laser (Spectra-Physics Beamlok, Santa Clara, CA, USA) that provided a $351-\mathrm{nm}$ emission source to excite an individual $\mathrm{ZnS}$ spring through the objective using a beam splitter, while the signal was collected by a charge-coupled device from the end of the spring.

\section{Fabrication and characterization of ZnS-microspring-based UV detectors}

ZnS-spring-based photosensors were fabricated using the following procedure. First, patterned $\mathrm{Cr} / \mathrm{Au}$ electrodes on $\mathrm{SiO}_{2} / \mathrm{Si}$ substrates were fabricated via UV lithography (Suss MicroTec, MA6/BA6, Garching, Germany) and an electron beam evaporation process (ULVAC, Kanagawa, Japan). Second, ZnS springs were suspended in ethanol via sonication and then placed on the electrodes. Finally, a focused ion beam (FIB; FB-2100, Hitachi, Tokyo, Japan) was used to deposit Pt onto the ends of ZnS springs to provide a good contact surface. The current versus voltages (that is, $\mathrm{I}-\mathrm{V}$ ) measurements of the light sensors were performed using a DC source (R 6144) to apply a bias and a picoammeter ( $\mathrm{R}$ $8340 \mathrm{~A})$ to measure the current. For the photodetection test, xenon calibrated by a UV-enhanced silicon photodiode provided incident light, while the timeresponse performance was recorded by a current meter after the illumination was switched off.

\section{RESULTS AND DISCUSSION}

The SEM image with a low magnification in Figure 1a shows the as-fabricated samples with an average length of several tens of micrometers, and these have a high density of springs. The typical morphology of a spring is shown in Figure $1 \mathrm{~b}$. When $\mathrm{Mn}$ is not present, the products can be characterized as winding wires (Supplementary Figure S1). Conversely, the products of springs can be fabricated under a thin $0.2-$ to $1-\mathrm{nm}$ film of $\mathrm{Mn}$ with a relatively stable yield. When the Mn thickness is increased above $1 \mathrm{~nm}$, the yield of the springs is lower; therefore, in this study, we used a $0.2-\mathrm{nm}$ film of $\mathrm{Mn}$ as a catalyst to produce springs with a 400 - to $800-\mathrm{nm}$ diameter, as shown in Supplementary Figure S2. Regarding the metal catalyst, the catalytic activities are likely influenced by the different 
valencies of the metal atoms and their electronic properties. ${ }^{14}$ Unlike $\mathrm{Au}$ that facilitates a traditional polar surface of [0001] for $\mathrm{ZnS}$ materials, $\mathrm{Mn}$ with its lower electronegativity (1.55) than $\mathrm{Au}$ (2.54) would lead to a different growth direction of the desired onedimensional structures. ${ }^{15}$ In addition, we found that a single spring could have different switching directions, as shown in Figure 1c. It should be noted that $\mathrm{ZnS}$ springs have both right- and left-handed chiralities. The Raman spectra (Figure 1d) of the proposed springs was measured at ambient conditions. The strong, first-order, scattering peak was centered at $346 \mathrm{~cm}^{-1}$, which can be indexed to A1 and E1, and belongs to the symmetric longitudinal optical (LO) modes of wurtzite ZnS materials. ${ }^{16}$ Simultaneously, a resolvable doublet was observed in the lower frequency region. Through a Lorentzian-line shape analysis, the results of which are shown in the inset of Figure 1d, this doublet centered at $269 \mathrm{~cm}^{-1}$ and $282 \mathrm{~cm}^{-1}$ can be identified as A1 $\left(269 \mathrm{~cm}^{-1}\right)$ and E2 $\left(282 \mathrm{~cm}^{-1}\right)$ of the transverse optical (TO) modes, respectively. ${ }^{17}$ For the peak at $216 \mathrm{~cm}^{-1}$, Ebisuzaki et al. assigned this broad feature to the first-order scattering of the longitudinal acoustic (LA) mode; however, more recent calculations indicate that this peak may come from acoustic phonon combination. ${ }^{18}$ Peaks in the range $350-450 \mathrm{~cm}^{-1}$ are frequently associated with a two-phonon density. ${ }^{19}$ For the proposed product, the broad peak at $\sim 640 \mathrm{~cm}^{-1}$ is due to the combination of the TO and LO modes, while the strong peak near $669 \mathrm{~cm}^{-1}$ may be relative to the $2 \mathrm{LO}$ overtones at some critical locations. ${ }^{18}$ To verify the distributions of the elements in the final products, energy-dispersive spectroscopy was performed on a single $\mathrm{ZnS}$ microspring, as shown in Supplementary Figure S3, which shows the characteristic peaks of the $\mathrm{S}$ and $\mathrm{Zn}$ elements without the peaks of the Mn catalyst; the Si and O signals originated from the growth substrate. The mapping results indicate that $\mathrm{Zn}$ and $\mathrm{S}$ are homogeneously distributed within a single ZnS spring, as shown in Figure 1e.

To determine the formation mechanism of $\mathrm{ZnS}$ springs, their intrinsic crystal structures were investigated via transmission electron microscopy (Figure 2a) with a uniform shape and contrast. As confirmed by high-resolution transmission electron microscopy images and the corresponding selected area electron diffraction patterns acquired from different positions (red circles in the Figure 2a), the $\mathrm{ZnS}$ microspring is shown to exhibit a wurtzite structure with an axial direction of [0001]. However, as shown in Figure $2 \mathrm{~b}$ and $\mathrm{c}$, respectively, the coiling part of the $\mathrm{ZnS}$ microwire shows growth along the [01-11] direction. It is important to note that the spring shows no distortion in the crystal lattice around the 'twist' point. In addition, the traces of the two different parts of a long spring (Supplementary Figure S4) show that the spring did not twist during the growth process. If the structure is projected along the [1-210] direction, as shown in Figure $2 \mathrm{~d}$, the $\pm(10-1-1)$ and $\pm(10-11)$ surfaces are shown in the polar direction, in addition to the typical $\mathrm{Zn}$ and $S$ terminated polar surfaces $\pm(0001)$. Thus, we can determine the coiling process for a single-crystal microwire that forms the spring structure shown in Figure 2e, where the basic building block is marked in blue. The direction of $\langle 0-111\rangle$ has six unanimous crystallographic originations: [01-11], [-1101], [-1011], [0-111], [1-101] and $[10-11]$. If we define the block height along [0001] to be $h$, then the pitch distance $(L)$ can be calculated by $L=6 h{ }^{20}$ The mean diameter $(D)$ of a single microspring, which is the average of the inner and outer diameters, can be deduced from the centers of the wires (vertical to their axial direction), which is approximately $D \approx 2 h / \tan \theta$, where $\theta$ is the angle between [01-11] and [0-110], as shown in Figure 2e. For the sample in Figure 2a, the spring has a mean diameter of $D \approx 480 \mathrm{~nm}$ and an average pitch distance of $L \approx 750 \mathrm{~nm}$. The derived angle based on $D$ and $L$ is $\theta \approx \arctan (L / 3 \mathrm{D})=27.51^{\circ} \pm 1^{\circ}$. The calculated result matches well with the expected inclination in the theory between the growth direction [01-11] and the c-plane (0001) for $\mathrm{ZnS}$ springs (for example, $\sim 62^{\circ}$, as calculated via the complementary angle relationship). In this case, we can produce the basic building block for the spring structure shown in Figure $2 \mathrm{f}$, where the growth direction of $\langle 0-111\rangle$ has six equivalent originations with a $60^{\circ}$ rotation between two adjacent directions. From the top view of a single spring (Figure 2g), the non-polar $(0-112)$ surface and $S^{2-}$ terminated (-110-1) and (10-1-1) surfaces are shown. Therefore, to form the self-coiling structure as in the model shown in Figure $2 \mathrm{~h}$, building blocks can be stacked along the [0001] axial direction that has six equivalent orientations without introducing deformation or twist. It is believed that the $\mathrm{Zn}$ terminated with the polar surface (0001) guides the wire growth through a self-catalysis process, while the new polar surfaces $\pm\{01-11\}$ drive the screw-coiling structure. Therefore, these $\mathrm{ZnS}$ springs were produced by rolling a single-crystal wire to reduce electrostatic energy, while their shape and dimensions were determined by the balance between the elastic deformation and the electrostatic interaction. ${ }^{21}$ Switching of the chirality of the springs is caused by the clockwise and/or counterclockwise stack direction of the blocks. The $c$ axis [0001] maintains the uniaxial direction during the growth of the microsprings that have no tendency toward either right- or left-handed chirality because the two polar surfaces have the same magnitude of electrostatic interaction energies but with opposite signs. ${ }^{22}$ These novel ZnS springs can provide insight into the growth process of wurtzite materials and help exploit their physical and optical properties when used in optoelectronic devices.

EBI could lead to direct interactions between incident electrons and a sample, which is an unavoidable process under SEM and transmission electron microscopy operations for material characterization and structural analysis. ${ }^{23}$ However, these effects on the samples may be either positive or negative and thus should be used appropriately to meet specific requirements. For this purpose, the influence of irradiation on the transport properties of individual $\mathrm{ZnS}$ microsprings were investigated in situ in an SEM chamber, as shown in Figure 3a with an SEM image in inset of Figure 3b. A typical I-V curve for the twoterminal structure under an EBI $(10 \mathrm{keV})$, as shown in Figure $3 \mathrm{~b}$, indicates that the irradiation-induced current can reach $9.5 \mathrm{pA}$ with a bias of $25 \mathrm{~V}$. It was also found that the dark current was beyond the current meter's detecting limit $\left(10^{-14} \mathrm{~A}\right)$; these results are in agreement with the published results in the literature. ${ }^{24}$ By switching the electron beam on and off, the device showed a good repeatability (Figure 3c) and fast response $(<1 \mathrm{~s})$, as determined from the enlarged portions of the rise and fall in Supplementary Figure S5. In this study, these twoterminal devices cannot be completely turned off; however, a residual current was detected as a result of the high-energy irradiation. ${ }^{25}$ Sensitivity $(R)$ can be deduced from the formula $R=\left(I_{\mathrm{on}}-I_{\mathrm{off}}\right) / I_{\mathrm{off}}$, where $I_{\text {off }}$ is the current measured by switching off the irradiation and $I_{\mathrm{on}}$ is the current induced by the electron beam with a bias of $25 \mathrm{~V}$. The variation of $R$ with the electron beam energy is plotted in Figure 3d. As the electron beam energy increases, $R$ tends to increase but then declines after a critical point near $5 \mathrm{keV}$. For a metal-semiconductormetal structure, such as that tested, the energy distributions of the carriers in the metal and semiconductor both govern the electron injection efficiency. For n-type semiconductors under EBI, the generated free electrons are controlled by the absorption/desorption of oxygen molecules, as in photo-carrier dynamics. ${ }^{26}$ Additional carriers in the $\mathrm{ZnS}$ springs and additional electrodes in the illumination area were produced via increased electron energy. As a consequence, it 

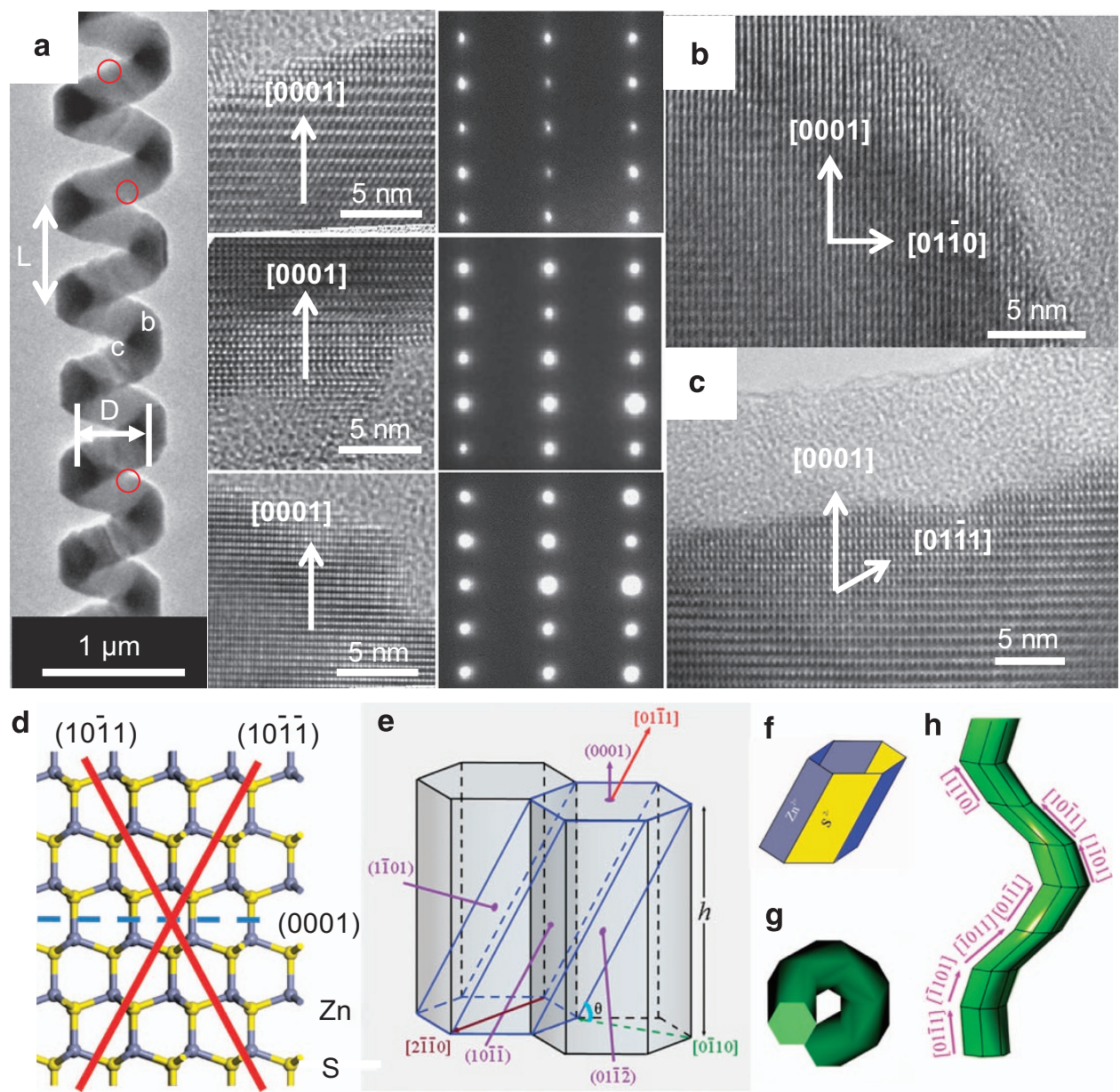

Figure 2 (a) Bright-field TEM image of a microspring with corresponding high-resolution TEM images and selected area electron diffraction (SAED) patterns; (b, c) TEM images recorded from positions b and $\mathbf{c}$ in a, respectively; (d) atomic structure of wurtzite ZnS along [1-210], showing the type polar surfaces of $\pm(0001)$ and $\{10-1-1\}$; (e) fundamental building block (in blue) of the microwire used in the spring structure with its growth direction and surfaces; (f) $\mathrm{Zn}^{-}$and $\mathrm{S}^{-}$surfaces for the building block; (g) top-down view and (h) a schematic model of the microspring with a symmetry of 6-fold right-hand screw rotation. TEM, transmission electron microscope.

is necessary to consider electron-electron interactions, such as Coulomb interaction and coherent scattering, for the electron transport in $\mathrm{ZnS}$ micro- or nano-structures. In this study, an effective capacitance model and a non-zero dwell time among the electron-electron interactions is assumed; thus, the conductance $(G)$ can be expressed by the formula: $\mathrm{dI} / \mathrm{dV}=G=G_{0}+\delta G$, where $G_{0}$ refers to a noninteracting part and $\delta G$ is attributed to the interaction correction that depends on the parameter $\beta$ in the formula $G / G_{0}=1-\beta+O(G / g)$, where $g=G_{0} h / e^{2}{ }^{27}$ Once the semiconductor is illuminated with an electron beam of a higher energy than the critical value, $\beta \rightarrow 1$; therefore, $\delta G$ becomes negative, and the relatively high-electron irradiation tends to suppress the conductance in the double-barrier structures. ${ }^{28}$ Motivated by the response to EBI, ZnS springs with a high crystallization, a well-defined geometry and a large surface-to-volume ratio would be particularly useful in optoelectronic devices. In the following paragraph, an initial investigation on their photo response is provided.

Owing to their self-coiling structure, a focused ion beam was used to fix a single spring on a substrate with electrodes that have a $10-\mu \mathrm{m}-$ long channel, as shown in the inset of Figure 4a. The remainder of Figure $4 \mathrm{a}$ shows the spectral selectivity of a two-terminal photodetector with a bias of $16 \mathrm{~V}$ over a wavelength range from 275 to
$550 \mathrm{~nm}$. A relatively low gain was observed when the illumination energy was below $450 \mathrm{~nm}$ but then increased up to $\sim 2$ orders of magnitude higher by adjusting the photon energy level to be above $335 \mathrm{~nm}$. For $\mathrm{ZnS}$ materials, photons with more energy than the semiconductor bandgap (that is, wavelengths $<335 \mathrm{~nm}$ for hexagonal phase $\mathrm{ZnS}$ ) can generate hole-electron pairs due to desorption of oxygen molecules. ${ }^{29}$ The system used to measure the photoresponses of an individual, ZnS-spring-based photodetector is shown in the inset of Figure $4 \mathrm{~b}$. The I-V characteristics of the photodetector (Figure $4 \mathrm{~b}$ ) were measured on these devices when exposed to 320-nm UV light with a bias of $16 \mathrm{~V}$ in air and exhibited a photocurrent up to $3 \mathrm{pA}$. The nonlinear behavior of the device's I-V curve may be associated with blocking at the metal/semiconductor contacts. Repeatability and response speed are the two key parameters to determine the capability of a light sensor. Over several cycles by turning the illumination on and off, the device (Figure $4 \mathrm{c}$ ) showed very good stability with a bias of $16 \mathrm{~V}$. This photodetector also showed a fast response that exceeded the measuring limit of the measurement system (that is, $0.3 \mathrm{~s}$ ) based on the enlarged portions in Supplementary Figure S6 (for example, the rising edge at 30-34s and the falling edge at 318-323 s). The quick and reversible switching between the illumination being on or off suggests that these sensors are potentially useful in advanced 
a
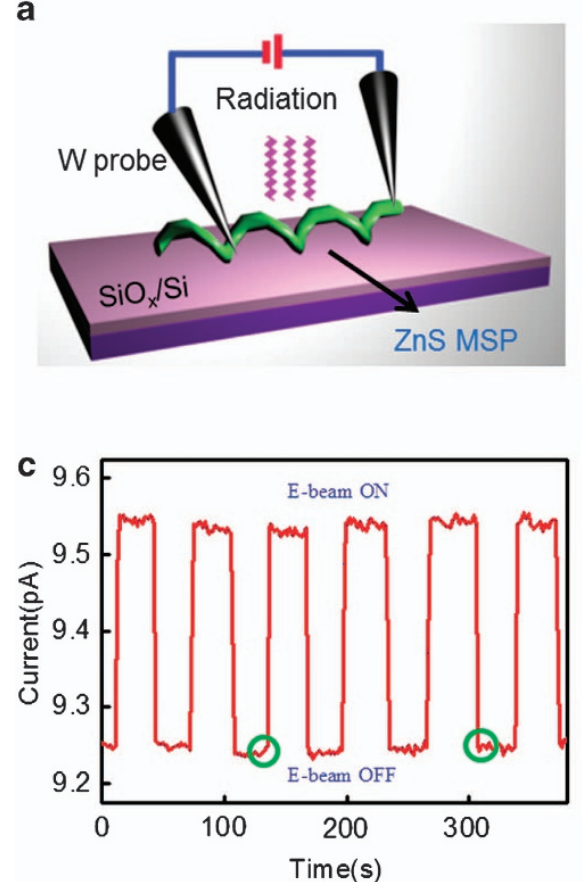

b
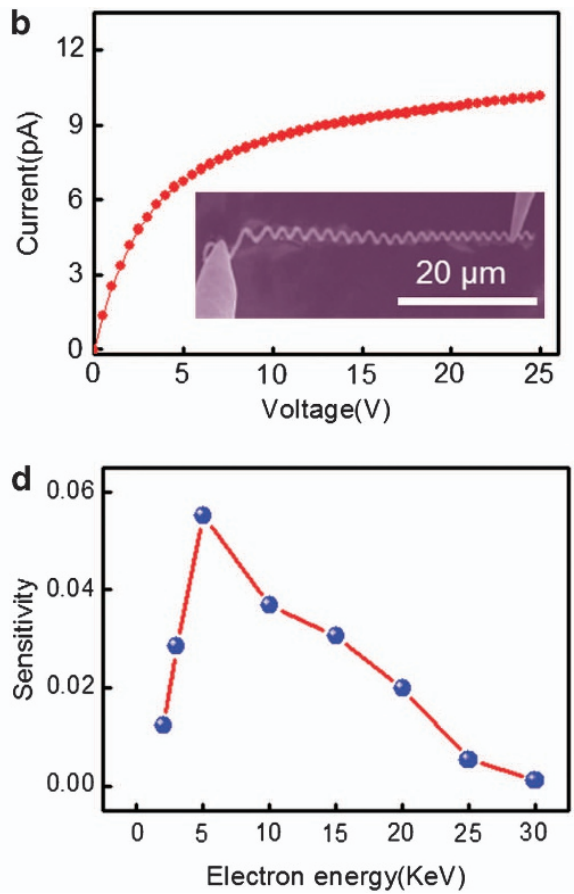

Figure 3 (a) Schematic diagram for the measurement system used; (b) I-V curve recorded on a single spring under EBI at $10 \mathrm{keV}$ with an inset showing an SEM image of a single ZnS microspring connected to two tungsten probes; (c) reproducible on/off switching tests with a bias of $25 \mathrm{~V}$; and (d) sensitivity as a function of the electron beam energy with a bias of $25 \mathrm{~V}$. EBI, electron beam irradiation; MSP, microspring.
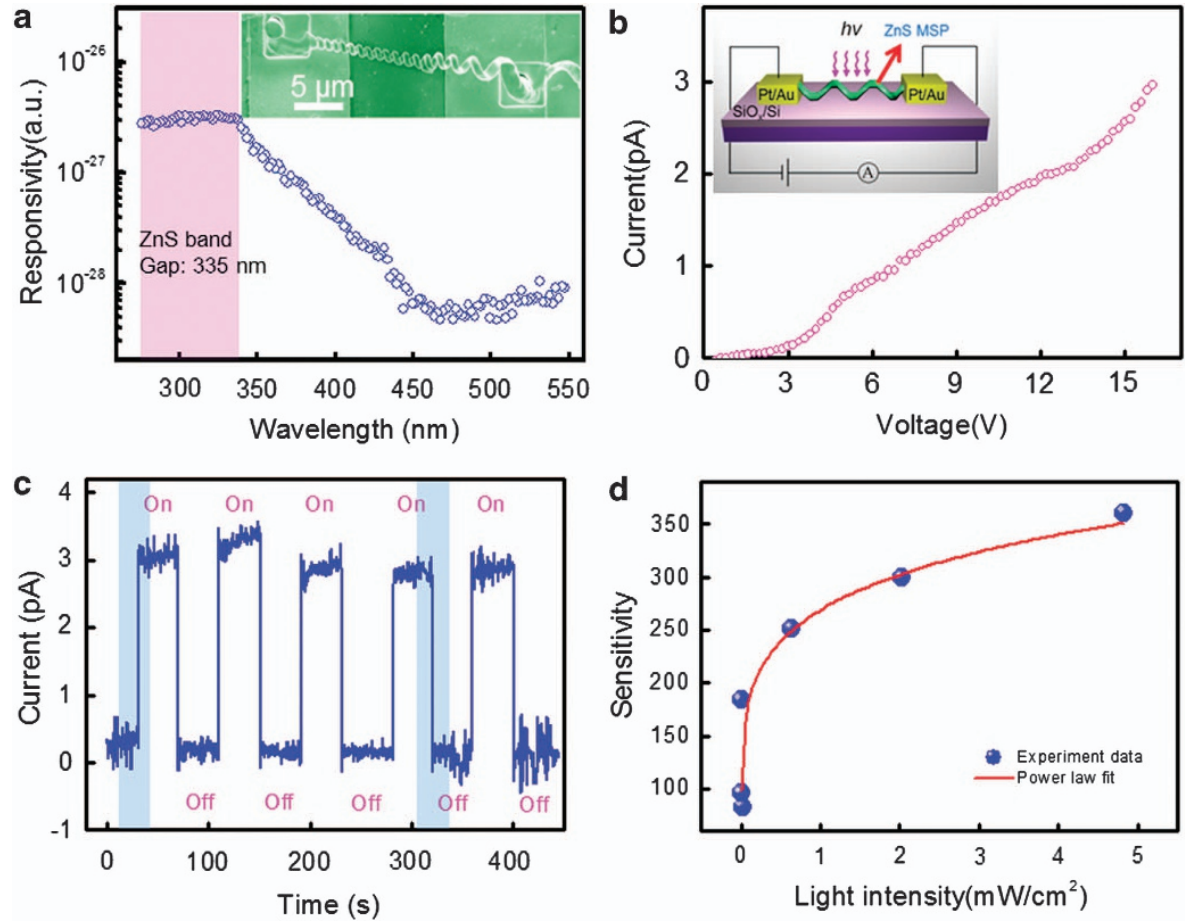

Figure 4 (a) Spectral selectivity for the UV light sensor with a bias of $16 \mathrm{~V}$ with an inset showing a representative SEM image of the sensor based on an individual ZnS microspring combining lithography and FIB; (b) I-V characteristics of a ZnS-spring photodetector exposed to a vertically illuminated UV light $(\sim 320 \mathrm{~nm})$ with a schematic diagram of the test system for an individual ZnS-spring-based photodetector; (c) reproducible on/off switching of the photo device with 320-nm light with a bias of $16 \mathrm{~V}$; and (d) response of the ZnS-spring photodetector at different light intensities at $320 \mathrm{~nm}$ and the corresponding fitting curve using the power law. a.u., arbitrary unit; FIB, focused ion beam; MSP, microspring; SEM, scanning electron microscope; UV, ultraviolet. 
photosensitive switches. For the photodetector investigated in this study, the current responsivity $\left(R_{\lambda}\right)$ was $\sim 0.02 \mathrm{~A} / \mathrm{W}$, and the calculated external quantum efficiency was $\sim 7.8 \%$ under $320 \mathrm{~nm}$ illumination at $2.04 \mathrm{~mW} \mathrm{~cm}^{-2}$ with an applied voltage of $16 \mathrm{~V}$. In addition, if considering the shot noise as the primary portion of the total noise, the detectivity $\left(D^{*}\right)$ in units of Jones, which is an index to evaluate the photoresponse, can be derived from $D^{*}=R_{\lambda} A^{1 / 2} /\left(2 e I_{\mathrm{d}}\right)^{1 / 2}$, where $I_{\mathrm{d}}$ is the dark current density. ${ }^{30}$ With the $320 \mathrm{~nm}$ UV light, the dark current of the ZnS-spring-based detectors was beyond the measurement range of the meter used (that is, $10^{-14} \mathrm{~A}$ ); thus, $D^{*}$ is larger than $9.52 \times 10^{8}$ Jones. In addition, the response of the ZnS-spring-based photodetector is dependent on the light intensity, as shown in Figure 4d; this phenomenon can be described by a simple power law, $R \sim P^{a}$, which yields a nearly linear behavior when $a \approx 0.2$, where $P$ is the power illumination. Such a non-unity exponent may be caused by a complex process of electron-hole generation, recombination and trapping within a semiconductor. ${ }^{31}$

Integrated optoelectronics are thought to be a potentially viable approach to mitigate restrictions, such as power dissipation, that hinder the further development of silicon-based electronic devices. ${ }^{32}$ The obtained $\mathrm{ZnS}$ springs provide an ideal system to investigate light propagation in a curved waveguide. In this study, as shown in Figure 5a, a 351-nm laser is focused on one end of an individual spring to generate luminescence signals and guide photons toward the other end of the waveguides, which are then collected by a chargecoupled device. Once the light propagates through the waveguides, intrinsic losses due to the absorption and scattering as a result of crystallite deformities or surface configuration are inevitable. ${ }^{33}$ To determine the propagation loss in individual ZnS springs, we modified the position of the laser on a sample and studied the variation of the output emission, as shown in Figure 5b. The bottom image is the corresponding dark-field optical image when the laser was focused on position 5. The collected spectra all exhibit a broad emission at $\sim 520 \mathrm{~nm}$ and a narrow emission centered at $450 \mathrm{~nm}$, as shown in Figure $5 c$, which can be attributed to either the zinc vacancies'activated defect centers or an energy transformation between zinc vacancies and sulfur vacancies. ${ }^{34}$ In this experiment, the $\mathrm{ZnS}$ springs have a three-dimensional helical structure compared with nanowires; consequently, the focal plane should be defocused at certain locations, leading to varying emission collection efficiencies at different excitation spots. Thus, some parts of the spectra may be darker. In a single microspring, as schematically shown in the inset of Figure $5 c$, the entering light (Point A) would be reflected onto the inner surface (Point $\mathrm{B}$ ) due to the different refractive indices between $\mathrm{ZnS}$ and the surroundings (that is, air in the experiments in this study). Thus, scattering could be observed in the zone between the two adjacent directions that are rotated at $60^{\circ}$ to each other (Point C). From the plot of the output emission intensity versus the propagation distance along the fiber of an individual $\mathrm{ZnS}$ microspring, the effective propagation length $L_{0}$, which is defined as the distance where the intensity decreases to $1 / e$ of its initial value, could be calculated via an exponential fitting: $I(x)=I_{0} \exp \left(-x / L_{0}\right)$, where $I_{0}$ is the initial intensity entering into the waveguide. For an emission at $\sim 520 \mathrm{mn}$ (Figure $5 \mathrm{~d}$ ), the propagation lengths were found to be $L_{0}=24.44 \mu \mathrm{m}$, which a
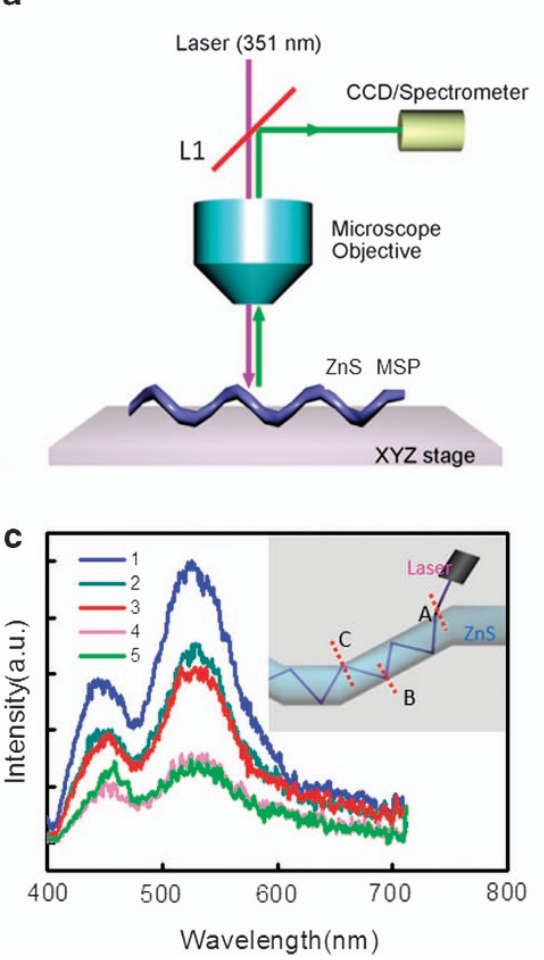
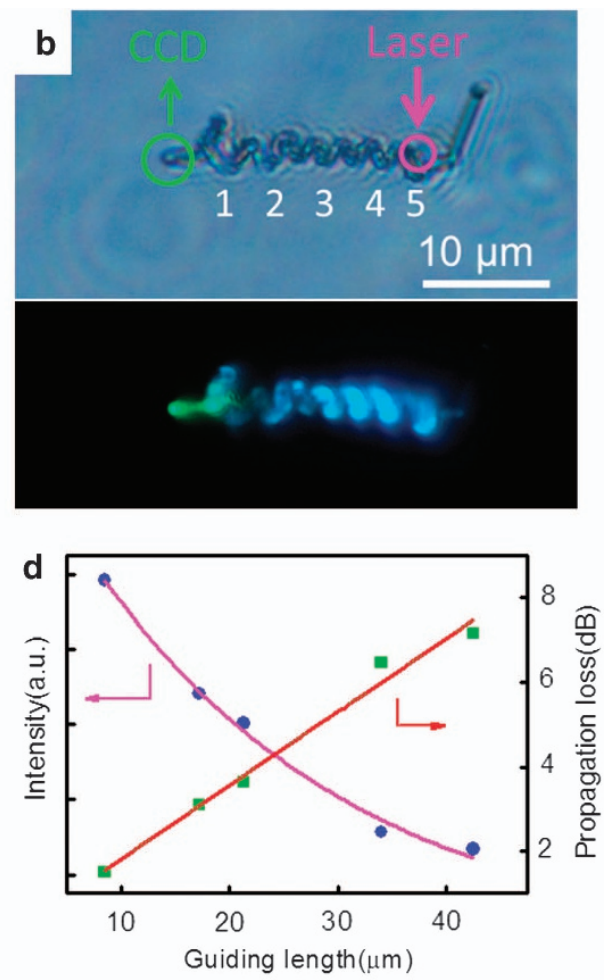

Figure 5 (a) Schematic representation of photoluminescence experiments, where L1 is a beam splitter; (b) bright field optical image and corresponding dark field images for a single ZnS microspring, where a 351-nm laser was focused on different positions to investigate the propagation loss of the waveguide; (c) corresponding photoluminescence spectra from different laser locations 1, 2, 3, 4 and 5 in $\mathbf{b}$ with an inset showing a schematic diagram of the light propagation in a ZnS microspring; (d) intensity of the output emission centered at $520 \mathrm{~nm}$ and the corresponding propagation loss for varying distances, where the purple curve is an exponential fitting of the emission intensity versus the propagation distance, and the red curve shows the linearly fitted propagation loss in $\mathrm{dB}$ as a function of the distance along the fiber of the microspring. a.u., arbitrary unit; CCD, charge-coupled device; MSP, microspring. 
corresponds to an attenuation coefficient $\alpha=1 / L_{0}=0.041 \mu \mathrm{m}^{-1}$. Based on the description of the loss in decibels $(\mathrm{dB})$, which is calculated by

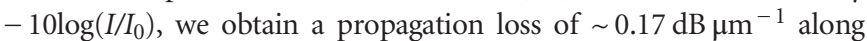
the fiber of the $\mathrm{ZnS}$ microspring, as shown by the red line in the figure. The total propagation loss for the spring in Figure $5 \mathrm{~b}$ with a fiber length of $\sim 59.52 \mu \mathrm{m}$ is $\sim 10.11 \mathrm{~dB}$. Thus, the propagation loss for

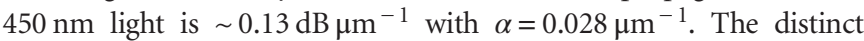
confinement characteristics for a special wavelength may be associated with the wavelength-dependent refractive indices for $\mathrm{ZnS}$ materials. In this study, it is found that the $\mathrm{ZnS}$ microsprings exhibit a low propagation loss comparable to previously reported silver nanowires $\left(0.41 \mathrm{~dB} \mu \mathrm{m}^{-1} \text { for } 785 \mathrm{~nm}\right)^{33}$, CdS nanowires $\left(0.026 \mathrm{~dB}_{\mu \mathrm{m}}^{-1}\right.$ for

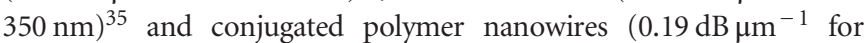
$295 \mathrm{~nm})^{36}$. The excellent light confinement of these novel structures indicates that leakage/scattering of light at the kinks has a negligible influence on the waveguide properties of $\mathrm{ZnS}$ microsprings, which can be attributed to the high-crystal quality of the as-synthesized products. ${ }^{37}$ Conversely, light absorption can be improved due to the multi-reflection of photons within $\mathrm{ZnS}$ microsprings. ${ }^{33}$ Therefore, the remarkable waveguide properties of $\mathrm{ZnS}$ microsprings indicate that they could be a promising candidate for future integrated photonics systems.

\section{CONCLUSION}

In summary, we synthesized deformation-free, single-crystal ZnS microsprings via vapor deposition and provided the first experimental evidence of their applications in optoelectronic devices. The growth of the $\mathrm{ZnS}$ springs is governed by the polar-surface-driven growth process, which is affected by a sequential change in the growth direction of $<0$ $111>$. The electrical characteristics of individual ZnS springs under EBI shows that a high-energy electron beam can produce higher gains, while the electron-electron interactions tend to suppress the conductivity of the springs. Photodetectors based on individual $\mathrm{ZnS}$ microsprings were successfully fabricated and underwent sensor evaluations, including light selectivity over a wavelength range and the response speed at a specific wavelength illumination were investigated. For light propagation in a single $\mathrm{ZnS}$ spring, it is shown that the light intensity followed an exponential decay along the fiber of a single $\mathrm{ZnS}$ microspring, which reveals an excellent waveguide performance with

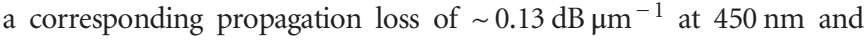
$\sim 0.17 \mathrm{~dB} \mu \mathrm{m}^{-1}$ at $520 \mathrm{~nm}$. The high-crystal quality, good response to UV light and excellent light confinement ability of $\mathrm{ZnS}$ springs indicate that they will likely be important in applications of future micro/ nanoscale optoelectronic systems.

\section{CONFLICT OF INTEREST}

The authors declare no conflict of interest.

\section{ACKNOWLEDGEMENTS}

This work was supported by the National Nature Science Foundation of China (21322106, 51472097 and 51402114), the Ministry of Science and Technology of China (2015CB932600) and the Fundamental Research Funds for the Central Universities. The authors would like to thank the Analytical and Testing Center of Huazhong University of Science and Technology.

1 Birowosuto, M. D., Yokoo, A., Zhang, G., Tateno, K., Kuramochi, E., Taniyama, H., Takiguchi, M. \& Notomi, M. Movable high-Q nanoresonators realized by semiconductor nanowires on a Si photonic crystal platform. Nat. Mater. 13, 279-285 (2014).

2 Xu, J. Q., Hou, G. H., Li, H. Q., Zhai, T. Y., Dong, B. P., Yan, H. L., Wang, Y. R., Yu, B. H., Bando, Y. \& Golberg, D. Fabrication of vertically aligned single-crystalline lanthanum hexaboride nanowire arrays and investigation of their field-emission behaviors. NPG Asia Mater. 5, e53. doi:10.1038/am.2013.25 (2013).

3 Oulton, R. F., Sorger, V. J., Genov, D. A., Pile, D. F. P. \& Zhang, X. A hybrid plasmonic waveguide for subwavelength confinement and long-range propagation. Nat. Photonics 2, 496-500 (2008).

4 Fu, Q., Zhang, Z. Y., Kou, L., Wu, P., Han, X., Zhu, X., Gao, J., Xun, J., Zhao, Q., Guo, W. \& Yu, D. Linear strain-gradient effect on the energy bandgap in bent CdS nanowires. Nano Res. 4, 308-314 (2011).

5 Fu, X. W., Liao, Z. M., Xu, J., Wu, X. S., Guo, W. L. \& Yu, D. P. Improvement of ultraviolet photoresponse of bent $\mathrm{ZnO}$ microwires by coupling piezoelectric and surface oxygen adsorption/desorption effects. Nanoscale 5, 916-920 (2013).

6 Duan, X. F., Huang, Y., Agarwa, R. \& Lieber, C. M. Single-nanowire electrically driven lasers. Nature 421, 241-245 (2003).

$7 \mathrm{Hu}$, Z. F., Guo, X. \& Tong, L. M. Freestanding nanowire ring laser. Appl. Phys. Lett. 103, 183104 (2013)

8 Zhang, C., Zou, C. L., Yan, Y. L., Wei, C., Cui, J. M., Sun, F. W., Yao, J. N. \& Zhao, Y. S Self-assembled organic crystalline microrings as active whispering-gallery-mode optical resonators. Adv. Opt. Mater. 1, 357-361 (2013).

9 Kong, X. Y., Ding, Y., Yang, R. S. \& Wang, Z. L. Single-crystal nanorings formed by epitaxial self-coiling of polar nanobelts. Science 303, 1348-1351 (2004).

10 Hughes, W. L. \& Wang, Z. L. Formation of piezoelectric single-crystal nanorings and nanobows. J. Am. Chem. Soc. 126, 6703-6709 (2004).

11 Song, K. W., Costi, R. \& Bulovic, V. Electrophoretic deposition of CdSe/ZnS quantum dots for light-emitting devices. Adv. Mater. 25, 1420-1423 (2013).

12 Fang, X. S., Zhai, T. Y., Gautam, U. K., Li, L., Wu, L. M., Bando, Y. \& Golberg, D. ZnS nanostructures: from synthesis to applications. Prog. Mater. Sci. 56, 175-287 (2011).

13 Moore, D., Ding, Y. \& Wang, Z. L. Hierarchical structured nanohelices of ZnS. Angew. Chem. Int. Ed. 45, 5150-5154 (2006).

14 Partenheimer, W. The unusual characteristics of the aerobic oxidation of 3,4-dimethoxytoluene with metal/bromide catalysts. Adv. Synth. Catal. 346, 1495-1500 (2004).

15 Martelli, F., Rubini, S., Piccin, M., Bais, G., Jabeen, F., De Franceschi, S., Grillo, V., Carlino, E., D'Acapito, F., Boscherini, F., Cabrini, S., Lazzarino, M., Businaro, L., Romanato, F. \& Franciosi, A. Manganese-induced growth of GaAs nanowires. Nano Lett. 6, 2130-2134 (2006).

16 Xiong, Q., Wang, J., Reese, O., Voon, L. C. L. Y \& Eklund, P. C. Raman scattering from surface phonons in rectangular cross-sectional w-ZnS nanowires. Nano Lett. 4, 1991-1996 (2004).

17 Schneider, J. \& Kirby, R. D. Raman scattering from ZnS polytypes. Phys. Rev. B 6 , 1290-1294 (1972).

18 Ebisuzaki, Y. \& Nicol, M. Raman spectrum of hexagonal zinc sulfide at high pressures. J. Phys. Chem. Solids 33, 763-766 (1971).

19 Arguello, C. A., Rousseau, D. L. \& Porto, S. P. S. First-order raman effect in wurtzite-type crystals. Phys. Rev. 181, 1351-1363 (1969).

20 Yang, R., Ding, Y. \& Wang, Z. L. Deformation-free single-crystal nanohelixes of polar nanowires. Nano Lett. 4, 1309-1312 (2004).

21 Kong, X. Y. \& Wang, Z. L. Spontaneous polarization-induced nanohelixes, nanosprings, and nanorings of piezoelectric nanobelts. Nano Lett. 3, 1625-1631 (2003).

22 Gao, P. X., Ding, Y., Mai, W. J., Hughes, W. L., Lao, C. S. \& Wang, Z. L. Conversion of Zinc oxide nanobelts into superlattice-structured nanohelices. Science 309, 1700-1704 (2005).

23 Zhang, Q., Qi, J. J., Li, X. \& Zhang, Y. Diameter-dependent internal gain in ZnO micro/ nanowires under electron beam irradiation. Nanoscale 3, 3060-3063 (2011).

24 Fang, X. S., Xiong, S. L., Zhai, T. Y., Bando, Y., Liao, M. Y., Gautam, U. K., Koide, Y., Zhang, X. G., Qian, Y. T. \& Golberg, D. Single-crystalline ZnS nanobelts as ultravioletlight sensors. Adv. Mater. 21, 2034-2039 (2009).

25 Colli, A., Fasoli, A., Beecher, P., Servati, P., Pisana, S., Fu, Y., Flewitt, A. J., Milne, W. I., Robertson, J., Ducati, C., De Franceschi, S., Hofmann, S. \& Ferraric, A. C. Thermal and chemical vapor deposition of Si nanowires: shape control, dispersion, and electrical properties. J. Appl. Phys. 102, 034302 (2007)

26 Peng, L., Hu, L. F. \& Fang, X. Low-dimensional nanostructure ultraviolet photodetectors. Adv. Mater. 25, 5321-5328 (2013).

27 Golubev, D. S. \& Zaikin, A. D. Coulomb interaction and quantum transport through a coherent scatterer. Phys. Rev. Lett. 86, 4887-4890 (2001).

28 Li, X., Qi, J. J., Zhang, Q., Wang, Z. Z., Lu, S. N. \& Zhang, Y. Investigation of electron beam detection properties of $\mathrm{ZnO}$ nanowire based back-to-back double Schottky diode. RSC Adv. 4, 12743-12747 (2014).

29 Zhai, T. Y., Fang, X. S., Liao, M. Y., Xu, X. J., Zeng, H. B., Bando, Y. \& Golberg, D. A comprehensive review of one-dimensional metal-oxide nanostructure photodetectors. Sensors 9, 6504-6529 (2009).

$30 \mathrm{Hu}$, P. A., Wang, L. F., Yoon, M., Zhang, J., Feng, W., Wang, X. N., Wen, Z. Z., Idrobo, J. C., Miyamoto, Y., Geohegan, D. B. \& Xiao, K. Highly responsive ultrathin GaS nanosheet photodetectors on rigid and flexible substrates. Nano Lett. 13, 1649-1654 (2013).

31 Li, L., Lee, P. S., Yan, C. Y., Zhai, T. Y., Fang, X. S, Liao, M. Y., Koide, Y., Bando, Y. \& Golberg, D. Ultrahigh-performance solar-blind photodetectors based on individual single-crystalline $\mathrm{In}_{2} \mathrm{Ge}_{2} \mathrm{O}_{7}$ nanobelts. Adv. Mater. 22, 5145-5149 (2010).

32 Barrelet, C. J., Greytak, A. B. \& Lieber, C. M. Nanowire photonic circuit elements. Nano Lett. 4, 1981-1985 (2004).

33 Wang, W. H., Yang, Q., Fan, F. R., Xu, H. X. \& Wang, Z. L. Light propagation in curved silver nanowire plasmonic waveguides. Nano Lett. 11, 1603-1608 (2011). 
34 Li, Z. P., Liu, B. B., Li, X. L., Yu, S. D., Wang, L., Hou, Y. Y., Zou, Y. G., Yao, M. G., Li, Q. J., Zou, B., Cui, T., Zou, G. T., Wang, G. R. \& Liu, Y. C. Synthesis of ZnS nanocrystals with controllable structure and morphology and their photoluminescence property. Nanotechnology 18, 255602 (2007).

35 Pan, A. L., Liu, D., Liu, R. B., Wang, F. F., Zhu, X. \& Zou, B. S. Optical waveguide through CdS nanoribbons. Small 1, 980-983 (2005).

36 Pyo, J., Kim, J. T., Yoo, J. \& Je, J. H. Light propagation in conjugated polymer nanowires decoupled from a substrate. Nanoscale 6, 5620-5623 (2014).

37 Piccione, B., van Vugt, L. K. \& Agarwal, R. Propagation loss spectroscopy on single nanowire active waveguides. Nano Lett. 10, 2251-2256 (2010). (c) (i) This work is licensed under a Creative Commons Attribution 4.0 International License. The images or other third party material in this article are included in the article's Creative Commons license, unless indicated otherwise in the credit line; if the material is not included under the Creative Commons license, users will need to obtain permission from the license holder to reproduce the material. To view a copy of this license, visit http:// creativecommons.org/licenses/by/4.0/

Supplementary Information accompanies the paper on the NPG Asia Materials website (http://www.nature.com/am) 